\title{
RESEARCH ON AIRCRAFT TARGET DETECTION ALGORITHM BASED ON IMPROVED RADIAL GRADIENT TRANSFORMATION
}

\author{
ZiMing Zhao, XueMei Gao, DanNi Jiang, YaoQiang Zhang* \\ Xi’an Surveying and Mapping Institute \\ Commission III, WG III/1
}

KEY WORDS: Aircraft Target Detection, Radial Gradient Transformation, Rotation-Invariant Feature, Polar Form, Look-up Table

\begin{abstract}
:
Aiming at the problem that the target may have different orientation in the unmanned aerial vehicle (UAV) image, the target detection algorithm based on the rotation invariant feature is studied, and this paper proposes a method of RIFF (Rotation-Invariant Fast Features) based on look up table and polar coordinate acceleration to be used for aircraft target detection. The experiment shows that the detection performance of this method is basically equal to the RIFF, and the operation efficiency is greatly improved
\end{abstract}

\section{INTRODUCTION}

The The target detection of unmanned aerial vehicle (UAV) image is mainly focused on how to automatically detect the object of interest from the UAV image. When the UAV executes the tasks just like military investigation or military attack, the targets of interest are usually vehicles, tanks, aircraft, bridges, oil depots and radar stations on the ground. For civil use, when the UAV goes on a patrol duty or other tasks, the main interest is the pedestrians, vehicles and so on.

The aircraft detection in UAV image has important military and civil needs, a large number of scholars have carried out an indepth study on this. Due to the arbitrary orientation of the aircraft in the real-time acquisition of UAV images, it is difficult to detect the target. The aircrafts in image have different orientation. There are some methods For the target detection, such as: The direction of the aircraft is estimating by the symmetry of the aircraft's fuselage. Multiple classifiers are training by target samples with different direction of aircrafts for the detection. The rotation invariant feature is extracting in the detection window.

In these above detection methods, the procedure of the aircraft orientation estimation and the training of multiple classifiers with different direction are complicated. When using the rotation invariant feature to detect, the feature extraction has problem of great computing, and the efficiency of the aircraft detection algorithm is reduced. For this reason, based on the RIFF using radial gradient transformation, a fast RIFF computing method based on look-up table and polar coordinates is proposed in this paper, which is applied to aircraft detection in UAV images.

\section{THE RIFF BASED ON RADIAL GRADIENT TRANSFORMATION}

The RIFF based on radial gradient transformation mainly includes two parts: the gradient projection and the region accumulation. The two parts guarantee the rotation invariance of feature. First starts with the gradient projection, i.e. the radial gradient transformation. As shown in Figure 1, the left is the feature extraction window before the target is rotated, and the right is the feature extraction window after the target is rotated in an angle $\theta$. The centre of the feature extraction window is expressed as $c$, and a point in the extraction window is $p$. The gradient vector of the point is expressed as $g$, and the radial local coordinate system of the point is recorded as pxy. The clockwise rotation matrix is $R_{\theta}$, and then the coordinate axis of the local coordinate system is expressed as follows:

$$
x=\frac{p-c}{\|p-c\|} ; y=R_{\pi / 2} x
$$

Then the expression of the gradient vector $\mathrm{g}$ under the radial local coordinate system pxy can be recorded as $\left(g^{T} x, g^{T} y\right)$.
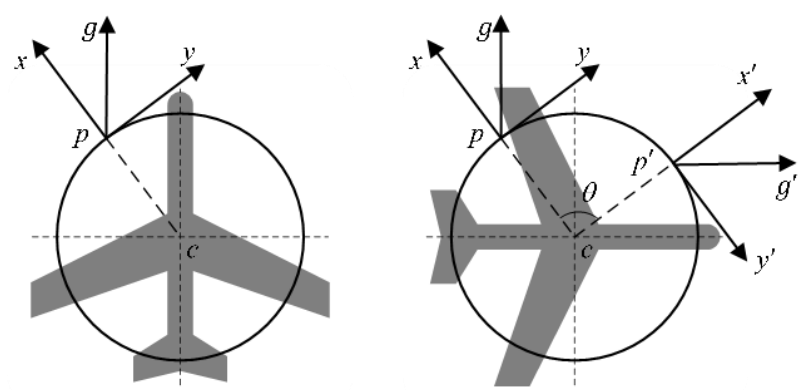

Figure 1. rotation invariance of radial gradient transformation

When the target is rotated a $\theta$ degrees clockwise, the $p$ is rotated to the new location as $p^{\prime}$, then the new radial local coordinate system is recorded as $p^{\prime} x^{\prime} y^{\prime}$, and the new local coordinate system satisfies the following formula:

$$
R_{\theta} p=p^{\prime} ; R_{\theta} x=x^{\prime} ; R_{\theta} y=y^{\prime} ; R_{\theta} g=g^{\prime}
$$


After the rotation the gradient vector $g^{\prime}$ of the new point $p^{\prime}$ in the radial local coordinate system $p^{\prime} x^{\prime} y^{\prime}$ can be recorded as $\left(g^{\prime T} x^{\prime}, g^{\prime T} y^{\prime}\right)$. The expression of the gradient vector in the radial local coordinate system is rotationally invariant, as shown below.

$$
\left(g^{\prime T} x^{\prime}, g^{\prime T} y^{\prime}\right)=\left(\left(R_{\theta} g\right)^{T} R_{\theta} x,\left(R_{\theta} g\right)^{T} R_{\theta} y\right)=\left(g^{T} x, g^{T} y\right)
$$

Radial gradient transformation can only ensure that the gradient vector is rotationally invariant in all local coordinate systems. Therefore, in order to extract the rotation invariant gradient feature of the detection window, the RIFF is used to calculate the gradient distribution using gradient accumulation based on the circular region as shown in Figure 2. No matter how the target rotates, the accumulation of gradient distribution in the radial local coordinates will remain the same. The RIFF describes the gradient distribution using the method shown in Figure 2.
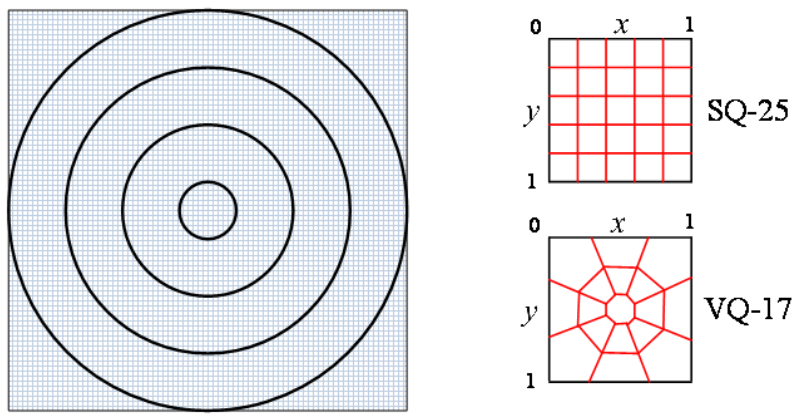

Figure 2. gradient accumulation in circular region

The two gradient quantization methods used in the RIFF are both two-dimensional histogram. The SQ-25 divides the gradient vector along the $x$ axis and the $y$ axis equally into 5 intervals. According to this method, a 25 dimensional gradient histogram can be getting through the gradient distribution in the statistical area. The VQ-17 divides the gradient direction into 8 intervals, and the gradient intensity into 3 intervals. According to this method, a 17 dimensional gradient histogram can be getting through the gradient distribution in the statistical area.

\section{FAST COMPUTING OF RIFF}

The calculation of the RIFF Based on radial gradient transformation is mainly reflected in the calculation of the radial local coordinate system and the projection operation of the gradient vector. As shown in Figure 3, the original coordinate system $x$ axis and $y$ axis is set to the row and column of the image along respectively. The gradient vector calculated on the original coordinate system is expressed as $g$, the radial local coordinate system is $o x^{\prime} y^{\prime}$, and the gradient vector represented in this coordinate system is $g^{\prime}$. Then the process of the radial gradient transformation can be simplified as expression (4).

$$
\left[\begin{array}{l}
g_{x}^{\prime} \\
g_{y}^{\prime}
\end{array}\right]=\left[\begin{array}{ll}
\cos \theta & \sin \theta \\
-\sin \theta & \cos \theta
\end{array}\right]\left[\begin{array}{l}
g_{x} \\
g_{y}
\end{array}\right]
$$

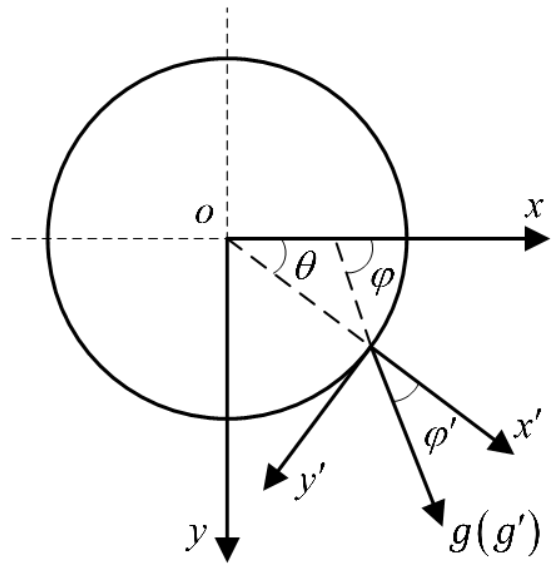

Figure 3. radial gradient transformation in polar form

The gradient vector $g$ and $g^{\prime}$ are expressed as $\left.\|g\|, \varphi\right)$ and $\left(\left\|g^{\prime}\right\|, \varphi^{\prime}\right)$ by being converted to polar form. The radial gradient transformation of Descartes coordinates in the form of (4) can be written as polar form, as follows:

$$
\left\{\begin{array}{l}
\left\|g^{\prime}\right\|=\|g\| \\
\varphi^{\prime}=\varphi-\theta
\end{array}\right.
$$

From the above formula, we know that the radial gradient transformation in polar form can avoid complex matrix operation in the form of Descartes coordinates by subtracting the current radial direction angle $\theta$ from the gradient direction $\varphi$. Although the transformation of coordinate conversion also needs root and arctangent operation, but when using a sliding window to extract dense RIFF in image, each pixel position only needs one conversion to avoid repeated calculations. In addition, the radial direction angle $\theta$ of each location in the feature extraction window can be stored in a lookup table in advance to avoid repeated calculations. Therefore, the radial gradient transformation based on look-up table and polar form proposed in this paper, when extracting dense RIFF, has less computation than radial gradient transformation (RGT) and approximate radial gradient transformation (ARGT), which will effectively improve the extraction efficiency of dense RIFF.

\section{EXPERIMENTAL RESULTS}

The experimental data set of aircraft detection includes 35 gray images with a resolution of $640 \times 480$ pixels, and the length of aircraft in the images changes from the range of 19 to 61 pixels. Three radial gradient methods are used to deal with each single frame, and the durations of consumption are shown in Table 1. The content just contains the durations of the radial gradient transformation, and does not contain the gradient quantization and the gradient accumulation process after the gradient transformation. In addition, the results of this method are given, as shown in Figure 4.

\begin{tabular}{|c|c|c|c|}
\hline methods & PRGT & RGT & ARGT \\
\hline durations & $373 \mathrm{~ms}$ & $711 \mathrm{~ms}$ & $884 \mathrm{~ms}$ \\
\hline
\end{tabular}

Table 1 consumption durations of three radial gradient methods 
The experimental result shows that compared with the radial gradient transformation (RGT) and approximate radial gradient transformation (ARGT), the radial gradient transformation based on look-up table and polar form (PRGT) can reduce the calculation of extraction and significantly improve the extraction efficiency of dense RIFF.
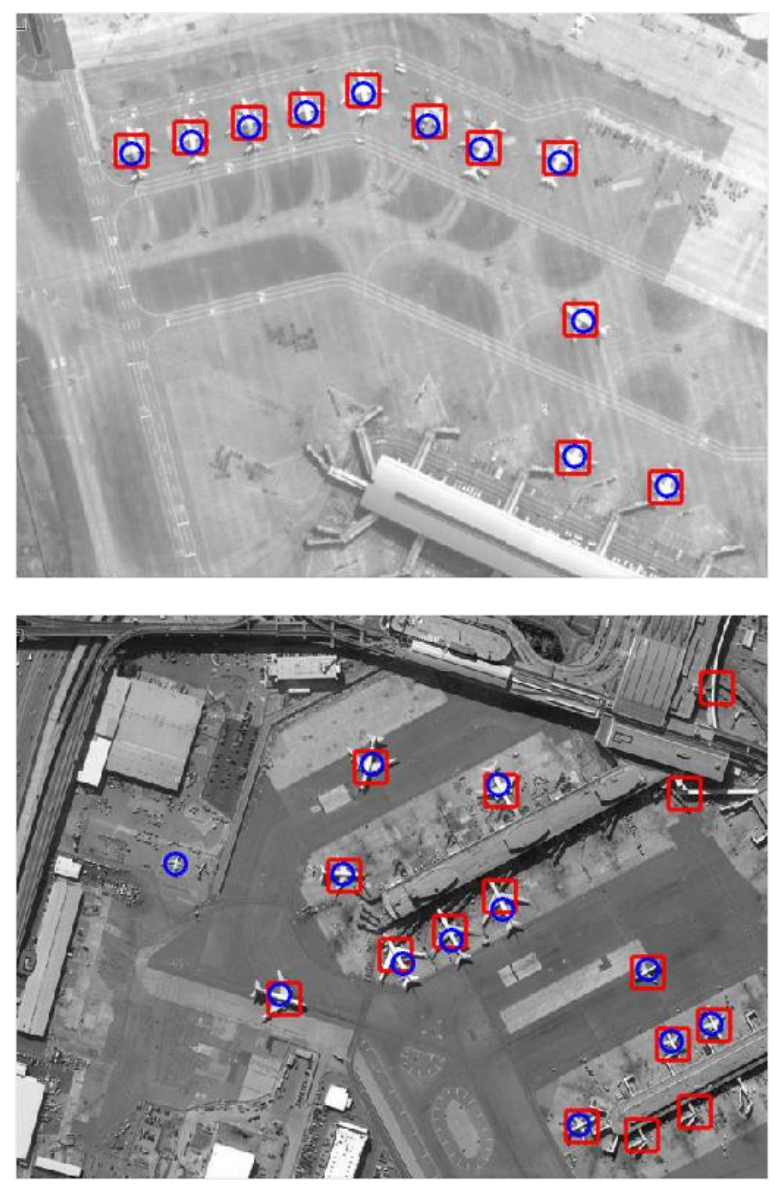

Figure 4 Experimental results

In Figure 4, the blue circles areas are hand-marked, and the Red Squares are the results detected by this algorithm. It can be seen from the Figure 4, the method proposed in this paper can be used to extract the aircraft target in the image very well. At the same time, for 35 test images, the detection rate of this algorithm is above $90 \%$, and the error rate is less than $10 \%$.

\section{CONCLUSION}

In the view of the shortcomings that the RIFF extraction based on the radial gradient transformation has a high time complexity, this paper proposes an improved fast radial gradient transformation method based on gradient look-up table and polar form. By comparison with the RIFF extraction by using RGT and ARGT, the extraction efficiency of the PRGT proposed in this paper has a significant improvement. Experimental result shows that the time consumption of the rotation invariant feature extraction is only half of the RGT and ARGT. Meanwhile, this method can achieve effective extraction of aircraft targets in UAV images.

\section{REFERENCES}

An Z, Shi Z, Teng X, et al. An Automated Airplane Detection System for Large Panchromatic Image with High Spatial Resolution. In: Optik, 2014, 125(12): pp. 2768-2775.

Chen X, Xiang S, Liu C, et al.2013. Aircraft Detection by Deep Belief Net. In: ACPR, IEEE. pp. 54-58.

Li W, Xiang S, Wang H, et al.2011. Robust Airplane Detection in Satellite Images. In: ICIP, IEEE. pp. 2821-2824.

Liu L, Shi Z. 2014. Airplane Detection Based on Rotation Invariant and Sparse Coding in Remote Sensing Images. In: Optik, 125(18): pp. $5327-5333$.

Sun H, Sun X, Wang H, et al. 2012.Automatic Target Detection in High-Resolution Remote Sensing Images Using Spatial Sparse Coding Bag-of-Words Model. In: IEEE Geoscience and Remote Sensing Letters, 9(1): pp. 109-113.

Takacs G, Chandrasekhar V, Tsai S, et al, 2010. Unified RealTime Tracking and Recognition with Rotation-Invariant Fast Features. In: CVPR, IEEE, pp. $934-941$. 